\title{
Three-dimensional evolution of magnetic and velocity shear driven instabilities in a compressible magnetized jet
}

\author{
Lapo Bettarini, ${ }^{1,2, a)}$ Simone Landi, ${ }^{2}$ Marco Velli, ${ }^{2,3}$ and Pasquale Londrillo ${ }^{4}$ \\ ${ }^{1}$ Katholieke Universiteit Leuven, Centrum voor Plasma Astrofysica, Celestijnenlaan 200B, \\ B-3001 Leuven, Belgium \\ ${ }^{2}$ Dipartimento di Astronomia e Scienza dello Spazio, Università degli Studi di Firenze, \\ Largo E. Fermi, 2, I-50125 Firenze, Italy \\ ${ }^{3}$ Jet Propulsion Laboratory, 4800 Oak Grove Drive, Pasadena, California 91109, USA \\ ${ }^{4}$ INAF Osservatorio Astronomico di Bologna, via C. Ranzani 1, I-40127 Bologna, Italy
}

(Received 12 January 2009; accepted 5 May 2009; published online 3 June 2009)

\begin{abstract}
The problem of three-dimensional combined magnetic and velocity shear driven instabilities of a compressible magnetized jet modeled as a plane neutral/current double vortex sheet in the framework of the resistive magnetohydrodynamics is addressed. The resulting dynamics given by the stream + current sheet interaction is analyzed and the effects of a variable geometry of the basic fields are considered. Depending on the basic asymptotic magnetic field configuration, a selection rule of the linear instability modes can be obtained. Hence, the system follows a two-stage path developing either through a fully three-dimensional dynamics with a rapid evolution of kink modes leading to a final turbulent state, or rather through a driving two-dimensional instability pattern that develops on parallel planes on which a reconnection+coalescence process takes place. (C) 2009 American Institute of Physics. [DOI: 10.1063/1.3142467]
\end{abstract}

\section{INTRODUCTION}

Velocity shears and strong magnetic field gradients are known to play an important role in the dynamics of both laboratory and astrophysical plasmas, such as solar flares, ${ }^{1,2}$ Earth's magnetotail, ${ }^{3,4}$ tokamaks, ${ }^{5}$ and, in the inner heliosphere, the interaction of the heliospheric current sheet with the structure determined by the slow component of the solar wind on the solar equatorial plane, embedded in the fast component. ${ }^{6-9}$ In fact, in a reference frame comoving with the slow wind, we have a bimodal flow profile where the velocity is zero at the heliospheric current sheet, and across it the interplanetary magnetic field changes sign from the southern to the northern solar hemispheres, regions of fast wind, resulting in a wake flow profile whose threedimensional (3D) evolution is not fully understood.

The incompressible two- and three-dimensional instability dynamics of a plane current vortex sheet have been largely investigated in previous works ${ }^{10,11}$ and only recently compressibility effects have started to be accounted at least for the linear regime. ${ }^{12}$ In Dahlburg et al. ${ }^{10}$ the simplest two-dimensional incompressible system is analyzed in the linear and nonlinear regime. The basic magnetic field and velocity field are both modeled by a hyperbolic tangent profile with a variable ratio of the velocity to the magnetic shear width, $\delta$, for different values of the Alfvènic Mach number, $\mathcal{M}_{a}$. The instability behavior of such systems depends strongly on these two parameters. In particular, for small values of $\mathcal{M}_{a}$ and large values of $\delta$, a direct energy transfer from the basic velocity field to the perturbed magnetic field is observed and, in general, the instability evolution cannot be considered simply as a mixture of the Kelvin-Helmholtz

${ }^{a)}$ Electronic mail: lapo.bettarini@ wis.kuleuven.be.
$(\mathrm{KH}$, hereafter) and resistive instabilities. The presence of a third direction is expected to modify the linear and the nonlinear regimes. First, it allows the ignition of all the instability modes of the system and, in particular, all the modes that are orthogonal to the basic flow; second, it allows the system to go from an initially laminar state to a turbulent one by means of a three-step process (a primary instability, e.g., the tearing mode for the neutral sheet, a two-dimensional quasisteady state, a further "secondary instability," caused by 3D disturbances). Such processes have been observed in several hydrodynamic and magnetohydrodynamic (MHD) configurations such as vortex ${ }^{13}$ and neutral sheets. ${ }^{14,15}$ Dahlburg and Einaudi ${ }^{11}$ considered an incompressible problem with a subsonic shear flow, whose profile is given by a hyperbolic tangent function with a width equal to that one of the neutral sheet. The perturbations they used consist of twodimensional disturbances of large amplitude, so that the system should be near the saturation condition in the nonlinear regime, with the addition of small amplitude 3D modes. The nonlinear evolution of those systems produces a complex structure wherein the 3D effects dominate with respect the two-dimensional evolution. The onset of the secondary instability has consequences also in the wake/jet dynamics. During the nonlinear stage of the resistive primary instability, the formation of magnetically confined plasma structures (plasmoids) is accompanied by the acceleration/deceleration of the central portion of the wake/jet. ${ }^{6,7}$ Once the secondary instability sets in, the free energy of the velocity shear triggers the transition toward a turbulent state and the acceleration/deceleration stops. ${ }^{6}$

Compressive effects have important consequences on the linear regime of the plane current-vortex sheet. Weakly evanescent modes are observed to grow and act also at large distances from the shear layers. Also, as shown in recent 


\section{clockwise rotation}

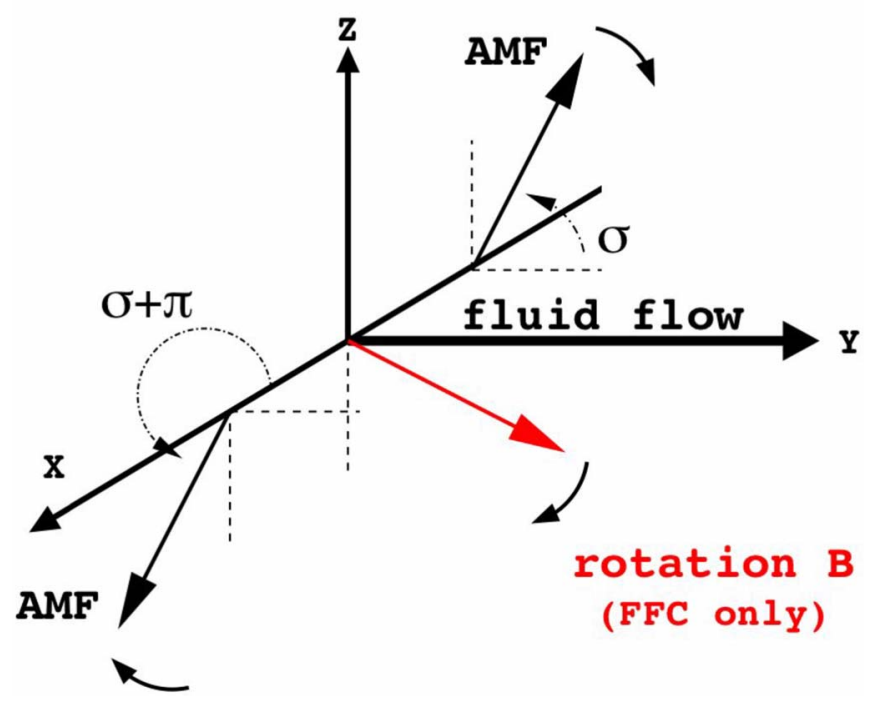

FIG. 1. (Color online) Reconstruction of the current/neutral double vortexsheet model used in the present work. The main features of the generic configuration are shown: the direction of the AMF, the type of rotation for the force-free magnetic field (clockwise or counterclockwise with respect to the rotation axis, $x$ ), and the presence (absence) of a magnetic field component within the fluid flow for the force-free (pressure balanced) equilibrium.

works on the $3 \mathrm{D}$ evolution of the compressive tearing instability, ${ }^{16,17}$ the onset of the secondary instability is observed to be critically dependent on the nature of the equilibrium, e.g., whether it is force free or pressure balanced, with or without a guide field.

If we consider a more complex two-dimensional system given by a jet/wake interacting with a current/neutral double vortex sheet in a compressible and magnetically dominated situation, ${ }^{9}$ the dynamics is observed to be dependent by the relative configuration of the basic magnetic to the velocity field, that is, as sketched in Fig. 1, by the angle $\sigma$ between the initial jet flow (or equivalently, the wake flow) direction and the asymptotic magnetic field (AMF, hereafter), that is the magnetic field "far enough" from the stream center. In particular, when $\sigma=\pi / 2$ in low beta configurations of supersonic but sub-Alfvénic shear flows (so, the AMF is orthogonal to the basic jet/wake flow), a typical KH instability fully develops and dominates the dynamics in the nonlinear regime. For any other angle in between $\sigma=0$ (the AMF being aligned to the jet/wake) and $\sigma<\pi / 2$, a linear varicoseresistive instability ${ }^{18}$ is dominating leading to magnetic field islands, which afterwards coalesce. In the present work, we extend the previous analysis to the $3 \mathrm{D}$ case both in the linear and nonlinear regimes.

In the following sections, we introduce the numerical setup, the initial conditions, the perturbations, and the parameters for our analysis (Sec. II). In Sec. III, we present the results of the simulations for the several different configurations under consideration. We will show how the details of the linear instability dynamics depend on the system's largescale structure, in particular, on the AMF direction, regardless of the type of equilibrium considered and how this drives into different dynamical paths in the nonlinear regime. In Sec. IV, we summarize the results drawing our conclusions and pointing to still open questions.

\section{EQUATIONS AND NUMERICAL SETTINGS}

We solve the set of one-fluid compressive-resistive MHD equations in Cartesian geometry. We define a streamwise, or Fourier, direction $(y)$ along which we impose periodic boundary conditions, a cross-stream direction $(x)$ along which the mean flow varies and we impose reflecting boundary conditions, and a spanwise direction (z), corresponding to an invariance direction for the quantities describing our initial system and along which we impose periodic boundary conditions.

In a compressible MHD system where high Mach number flows are supposed to form and afterwards undergo resistively triggered instabilities, the numerical strategy is a critical choice in order to obtain the best results in tracking the physical details of the expected dynamics. On the one hand, the understanding of dissipative processes such as magnetic reconnection requires high-order spectral-like numerical techniques such that the physical magnetic diffusion can be fully appreciated against the numerical dissipation. Furthermore, high-order methods allow to track the energy spectrum cascade toward high wave vectors with a reasonable number of grid points. On the other hand, supersonic flows can arise either in the initial state of the system or as a result of the reconnection process in a low plasma beta regime, and they determine plasma and magnetic field discontinuities that can be properly treated by means of shockcapturing techniques.

Here, we use the Eulerian conservative high-order code (hereafter, ECHO) to solve the full set of compressible and resistive MHD equations in a conservative form within the upwind constrained transport framework, ${ }^{19,20}$ which properly implements the magnetic field divergence-free condition. In general, ECHO solves the one-fluid equations for a magnetized plasma in different approximations, ranging from the special and general relativistic MHD frameworks ${ }^{21,22}$ to situations wherein the presence of the magnetic field diffusivity is taken into account. ${ }^{17} \mathrm{ECHO}$ is able to handle different high-order numerical techniques for flux reconstruction and computation of the derivatives. ${ }^{17,22}$ In particular, it allows the use of compact (or implicit) algorithms, ${ }^{23}$ which have spectral-like resolution properties better than the corresponding explicit numerical techniques. ${ }^{17}$

The set of equations to be solved is the following:

$$
\begin{aligned}
& \partial_{t} \rho=-\nabla \cdot(\rho \mathbf{v}), \\
& \partial_{t}(\rho \mathbf{v})=-\nabla \cdot(\rho \mathbf{v} \mathbf{v}+\mathcal{P} \mathbb{I}-\mathbf{B B}), \\
& \partial_{t} e=-\nabla \cdot[\mathbf{v}(e+\mathcal{P})-\mathbf{B}(\mathbf{v} \cdot \mathbf{B})-\eta \mathbf{B} \times \boldsymbol{\nabla} \times \mathbf{B}], \\
& \partial_{t} \mathbf{B}=-\nabla \times \mathbf{E}, \\
& \mathbf{E}=-\mathbf{v} \times \mathbf{B}+\eta \boldsymbol{\nabla} \times \mathbf{B},
\end{aligned}
$$

where $\rho$ is the mass density, $\mathbf{v}$ is the fluid velocity, 
$e=p /(\gamma-1)+\rho|\mathbf{v}|^{2} / 2+|\mathbf{B}|^{2} / 2$ the total (=hydro- plus magnetic) energy density according to a perfect gas equation of state, where $p$ is the plasma pressure and $\gamma$ is the adiabatic index; $\mathcal{P}=p+|\mathbf{B}|^{2} / 2$ is the total pressure. In Eqs. (3) and (5), an explicit dissipative term due to the plasma resistivity, $\eta$, is present though maintaining the same formal structure of the conservative framework.

In order to obtain the dimensionless equations, we use the characteristic quantities $\widetilde{L}, \widetilde{\rho}$, and $\widetilde{v}_{a}$, which correspond, respectively, to the velocity shear width, the initial uniform density, and the Alfvén velocity at the cross-stream boundaries. Time, velocity, and magnetic field strength are expressed in units of the related quantities $\widetilde{t}, \widetilde{v}$, and $\widetilde{B}$,

$$
\tilde{t}=\frac{\widetilde{L}}{\widetilde{v}_{a}}, \quad \widetilde{v}=\mathcal{M}_{a} \widetilde{v}_{a} \quad \widetilde{B}=\widetilde{v}_{a} \sqrt{4 \pi \widetilde{\rho}},
$$

with $\mathcal{M}_{a}$ being the Alfvénic Mach number, as already mentioned in the introduction. Moreover, plasma pressure and magnetic diffusivity are measured in terms of $\widetilde{p}=\beta \widetilde{B}^{2} / 2$ and of the inverse of the Lundquist number $\mathcal{S}=\widetilde{v_{a}} \widetilde{L} / \eta$.

\section{A. Simulation setup}

In the present work, two different configurations are considered: a force-free (FF, hereafter) and a pressure balanced (PB, hereafter) initial equilibrium. As shown in Fig. 1, the FF current sheet is formed by a rotation of the cross-sheet component of the magnetic field across the flow, whereas in the PB case no rotation is considered and the polarity reversal is obtained by means of a neutral sheet. So, FF and PB cases differ essentially due to the presence of the rotation component of the magnetic field along the flow (defined as the streamwise direction), which is absent for $\sigma=0$ (being directed along the orthogonal or spanwise direction) and becomes more and more relevant as the angle increases. Furthermore, for $\sigma=0$ and $\pi / 2$, the FF is intrinsically symmetric with respect to the streamwise direction, while this system does not have a defined symmetry at all for $0<\sigma<\pi / 2$. For the PB configuration, the system is always symmetric and in correspondence of the jet/wake we always have a neutral line. This initial parity property of the two configurations determines the evolution of the current and neutral sheet, since in the FF we have a preferential side for magnetic islands formation and a differential deceleration of the jet. The maximum distortion effect is observed for $\sigma=3 \pi / 8$. ${ }^{9}$

The FF basic fields are the following:

$$
\begin{aligned}
& v_{0 y}(x)=\mathcal{M}_{a} \operatorname{sech}^{2}(x), \\
& B_{0 y}(x)=[\sin \sigma \operatorname{sech}(\delta x)+\cos \sigma \tanh (\delta x)], \\
& B_{0 z}(x)=[-\cos \sigma \operatorname{sech}(\delta x)+\sin \sigma \tanh (\delta x)] .
\end{aligned}
$$

As already pointed out, current-stream interaction driven instabilities may have an important role in the dynamical evolution of several astrophysical plasma structures characterized by a low $\beta$ regime. For instance, the slow wind acceleration region above the sun's helmet streamers is characterized by a typical Alfvén speed less than $750 \mathrm{~km} \mathrm{~s}^{-1},{ }^{24}$ a sound speed of about $100 \mathrm{~km} \mathrm{~s}^{-1}$ and a typical differential velocity of fast and slow streams of about $300-400 \mathrm{~km} \mathrm{~s}^{-1}$. For our simulations, we consider the following general settings: the Alfvénic Mach is $\approx 0.73$, the sonic Mach number is equal to 3 , and so we have a supersonic and subalfvénic current+flow system characterized by a $\beta$ of 0.07 . As already pointed out, the angle $\sigma$ defines the initial direction of the AMF relative to the basic flow, having $\sigma=0$ when the AMF is parallel to the basic velocity field and $\sigma=\pi / 2$ when it is orthogonal to the velocity field. The width of the jet/ wake, $a_{v}$, provides the reference length to set the MHD equations dimensionless, $\tilde{L}$, and hence the dynamic time is $\tilde{t}=a_{v} / v_{a}$, while $\delta=a_{v} / a_{b}$ measures the ratio between the jet/ wake shear width and the current sheet width $\left(a_{b}\right)$. We consider the perfect gas equation of state $p_{0}=\rho_{0} T_{0}$ that initially gives

$$
\begin{aligned}
& \rho_{0}=\text { constant }=1.0 \\
& T_{0}=c_{0 s}^{2} / \gamma=\text { squared sound speed, }
\end{aligned}
$$

and we assume a polytropic equation with $\gamma$ equal to $5 / 3$.

The PB basic fields are given by the following relations:

$$
\begin{aligned}
& v_{0 y}(x)=\mathcal{M}_{a} \operatorname{sech}^{2}(x), \\
& B_{0 y}(x)=\cos \sigma \tanh (\delta x), \\
& B_{0 z}(x)=\sin \sigma \tanh (\delta x) .
\end{aligned}
$$

Pressure equilibrium condition is here assured by a gradient in the temperature profile,

$$
T_{0}=\frac{1}{2}\left(1+\beta_{\infty}\right)-\frac{1}{2}\left(B_{0 y}^{2}+B_{0 z}^{2}\right),
$$

where $\beta_{\infty}$ is the plasma beta at the cross-stream boundary for $t=0$.

The value of $\delta$ is observed to be a critical parameter for the evolution of the above-defined systems and we assume it equal to 10 . Such quite high value determines a strong gradient in the components of the basic magnetic field and this produces the conditions for an effective instability-triggering mechanism ${ }^{9,10}$ ). Besides, from Eqs. (6)-(8) and (11)-(13), we can observe that varying the angle $\sigma$ produces a rotation in the AMF. So, strong initial magnetic field gradients can affect in a nontrivial way the evolution of the system according to the chosen initial configuration, both in the direction determined by the jet/wake and in the orthogonal one.

We use two different sets of initial perturbations consisting in a proper two-dimensional space forcing on the crossstream component of velocity in the jet/wake plane,

$$
v_{1 x}(x, y, z)=\varepsilon \mathcal{F}(x) \sin \left(\frac{2 \pi l}{L_{y}} y+\frac{2 \pi m}{L_{z}} z+\phi_{r}\right),
$$

with $(l, m)$ being the excited modes in $y$ and $z$, respectively, with $l \in[0,12]$ and $m \in[-6,6], \phi_{r}$ a random phase, and $\varepsilon$ the initial perturbation amplitude. In Eq. (15), we set 


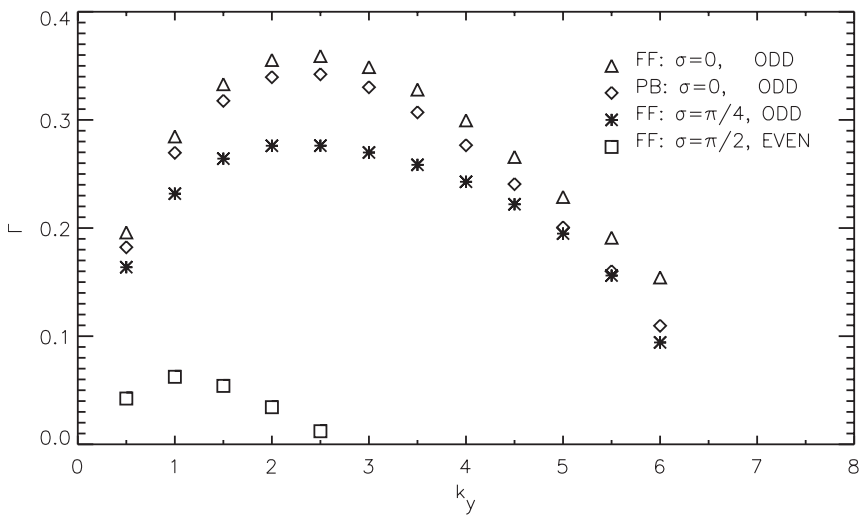

FIG. 2. Two-dimensional dispersion relations obtained by a linear twodimensional code for some of the PB and FF cases under investigations. For the FF " $\pi / 2$ " case, the system was perturbed by the even function (17) instead of the odd function (16).

$$
\mathcal{F}(x)=2 \tanh (\delta x) \operatorname{sech}(\delta x),
$$

or

$$
\mathcal{F}(x)=\operatorname{sech}(\delta x)
$$

in order to force the system with a function whose parity properties should resemble that of the dominating instability.

We use a 3D uniform grid with resolution $\left[n_{x}, n_{y}\right] \otimes n_{z}$ $=(480,120,30)$ with the following dimensionless sizes:

$$
\begin{aligned}
& L_{x}=\mathcal{D}_{x}[-2 \pi, 2 \pi], \\
& L_{y}=k_{\min }^{-1}[0,2 \pi], \\
& L_{z}=\mathcal{D}_{z}[0,2 \pi],
\end{aligned}
$$

where $\mathcal{D}_{x}$ and $\mathcal{D}_{z}$ are coefficients chosen to properly adapt the size of the grid along the $x$ and $z$ directions, respectively, while $k_{\min }$ is the smallest perturbation wave number along the $y$ direction, which sets the largest wavelength we can consider. The values of $L_{x}$ and $N_{x}$ have been chosen in order to have the cross-stream boundaries located sufficiently far from the current sheet in order to prevent stabilizing effects from the walls, but at the same time their distance must still allow us to resolve the field gradients within the current sheet with a reasonable number of grid points. A reasonable value of $N_{z}$ is chosen to follow the dynamics both in the linear and in the nonlinear regimes. Higher-resolution test simulations (up to double $N_{z}$ ) did not show sensitive differences both in the spectral behavior as well as in real-space dynamics. For what regards the streamwise direction, it must be chosen such to allow us to observe in the nonlinear regime both the direct cascade toward higher wave vectors and an inverse cascade toward large scale by means of a coalescence process. We verified our choice of the main parameters $\left(M_{a}, \delta, \beta\right)$ by means of a two-dimensional linear code whose details are reported in Ref. 17: the maximum linear growth
TABLE I. Summary of the performed 3D simulations. In the first column, we point out the two possible basic configurations: PB stands for pressure balance equilibrium and FF stands for force-free equilibrium. In the second column, we report considered values of the angle $\sigma$. For all simulations the following parameters are used: $\delta=10, \beta \approx 0.07, \mathcal{M}_{s}=3, \mathcal{M}_{a} \approx 0.7, \mathcal{S}$ $=2000$.

\begin{tabular}{cc}
\hline \hline Basic configuration & $\sigma$ \\
\hline $\mathrm{PB}, \mathrm{FF}$ & 0 \\
$\mathrm{~PB}, \mathrm{FF}$ & $\pi / 8$ \\
$\mathrm{~PB}, \mathrm{FF}$ & $\pm \pi / 4$ \\
$\mathrm{~PB}, \mathrm{FF}$ & $3 \pi / 8$ \\
$\mathrm{~PB}, \mathrm{FF}$ & $\pi / 2$ \\
\hline \hline
\end{tabular}

rates correspond to wave vectors in the range $[1,3]$ (see Fig. 2 ). We choose hence the following set of parameters for the numerical domain:

$$
\delta=10 \Rightarrow\left\{\begin{array}{l}
\mathcal{D}_{x}=0.75 \Rightarrow L_{x}=[-3 \pi / 2,3 \pi / 2], \\
k_{\min }=0.5 \Rightarrow L_{y}=[0,4 \pi], \\
\mathcal{D}_{z}=1 \Rightarrow L_{z}=[0,2 \pi] .
\end{array}\right.
$$

So, this streamwise size implies that the wave vector corresponding to the above mentioned maximum linear growth rate should correspond to a wave number $l$ in a range of $[2,6]$.

The simulations are performed by means of a seventh order compact scheme in order to have a low numerical dissipation influencing the expected resistivity-driven linear dynamics; we consider a Lundquist number equal to 2000. As in Landi et al. ${ }^{17}$ we verified that the intrinsic numerical dissipation, implicitly introduced by the adopted numerical scheme, is much lower than the explicit diffusivity assumed. In particular, by setting $n_{z}=1$ and canceling out the equilibrium magnetic field diffusion, we verified that the twodimensional linear evolution in ECHO is in very good agreement with the simulations performed by using the linear code.

In Sec. III we present results of the 3D instability both in the linear (Sec. III A) and nonlinear (Sec. III B) regimes. To study the linear regime, as for the two-dimensional simulations, the equilibrium magnetic field diffusion, which affects the growth rates of the linear modes, ${ }^{17}$ is cancelled out. On the contrary, the study of the nonlinear regime has been performed by including the effect of the diffusion of the equilibrium magnetic field. In Table I, we report the simulations analyzed in the paper.

\section{RESULTS ON THE 3D INSTABILITY}

We consider several values of $\sigma$ for both the FF and PB configurations, also for continuity reasons with the twodimensional analysis ${ }^{9}$ of these configurations: $0, \pi / 8, \pm \pi / 4$, $3 \pi / 8$, and $\pi / 2$. Particular attention must be given to the FF cases with $\sigma=3 \pi / 8$ in correspondence of which it is observed a peculiar two-dimensional dynamics characterized by a maximum effect in the asymmetries introduced by magnetic field basic configuration. ${ }^{9}$ 


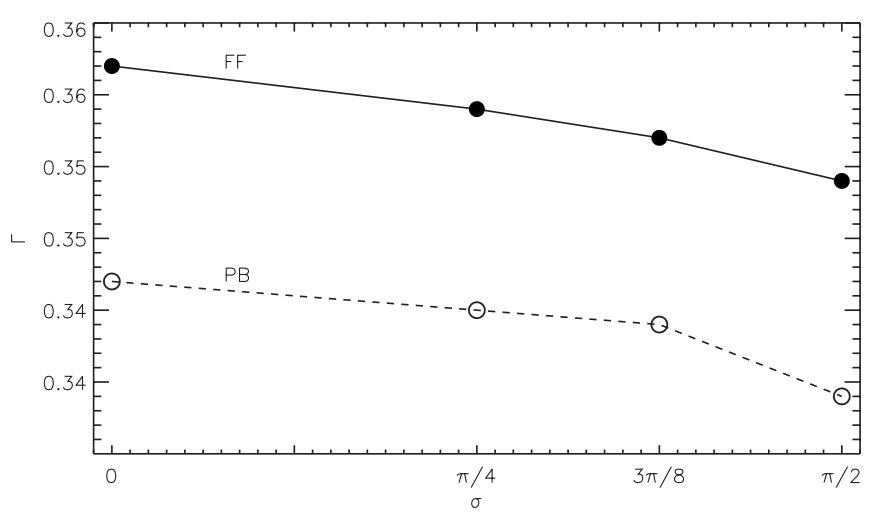

FIG. 3. Maximum growth rate as a function of $\sigma$ for the FF (solid line) and $\mathrm{PB}$ (dashed line) configurations, during the linear regime of the 3D simulations.

\section{A. Linear regime}

Consider the results from a two-dimensional analysis. For $\sigma<\pi / 2$ the varicose-resistive modes dominate the instability dynamics; ${ }^{10}$ a maximum growth rate of about $\Gamma \sim 0.35$ is attained for $\sigma=0$ decreasing as $\sigma$ increases. ${ }^{9}$ For $\sigma=\pi / 2$, the varicose-resistive modes are no longer unstable: the AMF is now orthogonal to the jet/wake hence it does not prevent the development of KH dynamics. ${ }^{9,25}$ In Fig. 2, we show the dispersion relation $\left(\Gamma\right.$ as function of $\left.k_{y}\right)$ for a few cases. The $\sigma=\pi / 2$ case refers to the sinuous modes given by Eq. (17) and it is evident that the growth rate of the $\mathrm{KH}$ instability $(\Gamma \sim 0.07)$ is much lower than those for the varicose-resistive modes with $\sigma<\pi / 2$.
In the 3D simulations, the situation changes significantly. In fact, as shown in Fig. 3, we obtain the same growth rates of about $\Gamma \approx 0.35$, regardless of the value of $\sigma$. Moreover, all the cases are characterized by the development of varicose-resistive modes, as we can infer from the fact that the growth rates match the values observed in the twodimensional linear simulations with odd perturbations. In general, the duration of the linear phase is slightly longer in the $\mathrm{PB}$ cases than in the FF ones since the growth rates of the fastest modes are a bit smaller. This is consistent with the 2D linear simulations. This different behavior is clearly related to the fact that in three dimensions all instability modes are allowed to develop. In Fig. 4, we report the magnetic energy spectrum in the Fourier space $k_{z}-k_{y}$ at a given time during the linear regime for the FF (upper row) and the PB (lower row) configurations. The cases with $\sigma=0,3 \pi / 8$, and $\pi / 2$ are shown in the left, middle, and right columns, respectively. As the instability dynamics sets in, both the FF and the PB configurations, where the AMF is aligned with the basic fluid jet $(\sigma=0)$, are driven mainly by the modes characterized by $k_{z}=0$, although a small amount of energy is present in modes with $k_{z}= \pm 1,2$, at least for the PB configuration. Consistently with the linear code results, The observed fastestgrowing linear mode lies between $k_{y}=2,2.5$. If now we consider a value of $\sigma$ between 0 and $\pi / 2$, such as $\sigma=3 \pi / 8$ shown in the middle panel of Fig. 4, we observe that the most unstable modes are no more aligned with the jet/wake flow. The instability grows along a preferential direction that is selected by the direction of the AMF, underlined in the figure by the dash-dotted line. The most unstable modes for
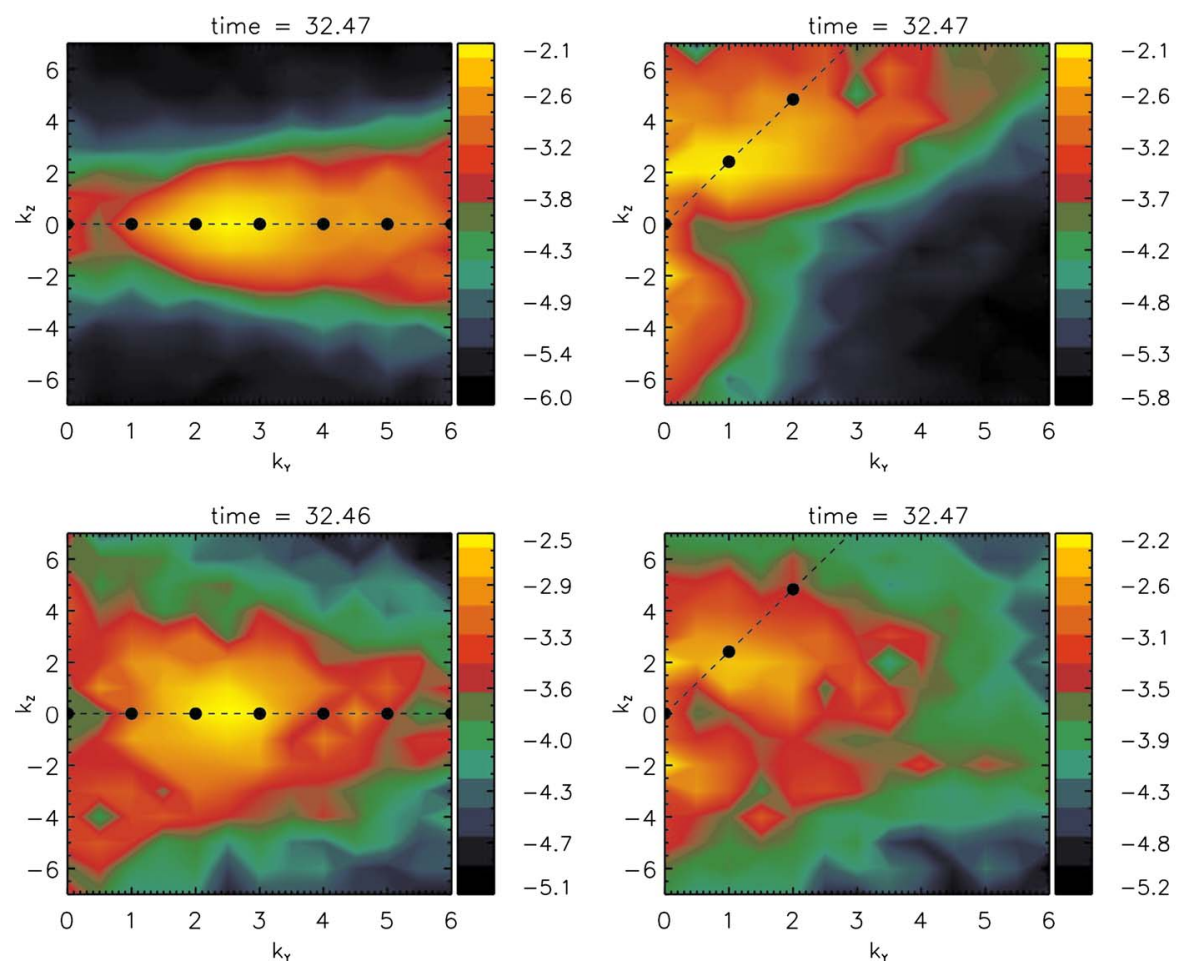
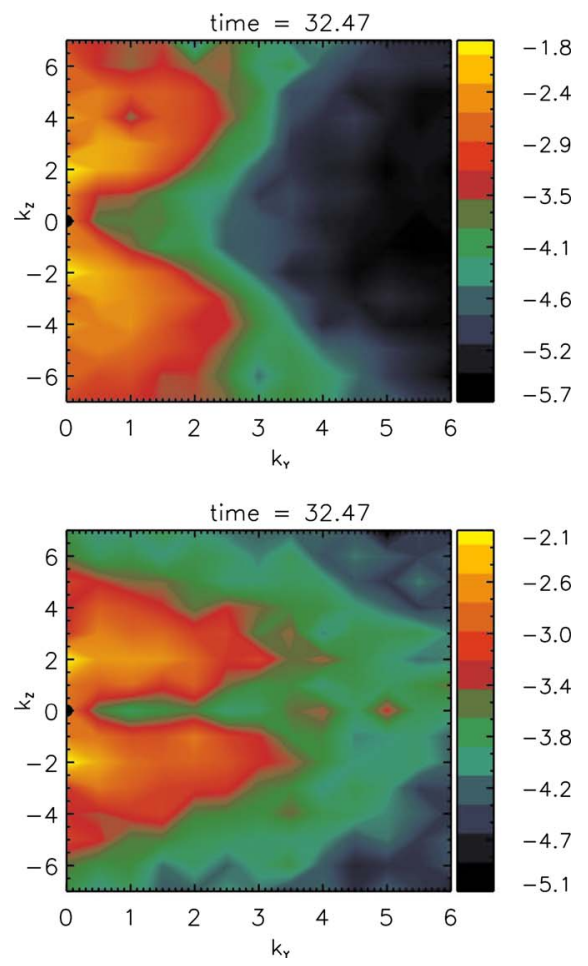

FIG. 4. (Color online) Magnetic energy (logarithmic scale) in the $k_{y}-k_{z}$ Fourier space for three different values of $\sigma(0$ in the left panel, $3 \pi / 8$ in the midpanel, and $\pi / 2$ in the right panel, respectively) for FF (upper row) and PB (lower row) at the end of the linear regime. The point-dashed line highlights the direction of the AMF. 

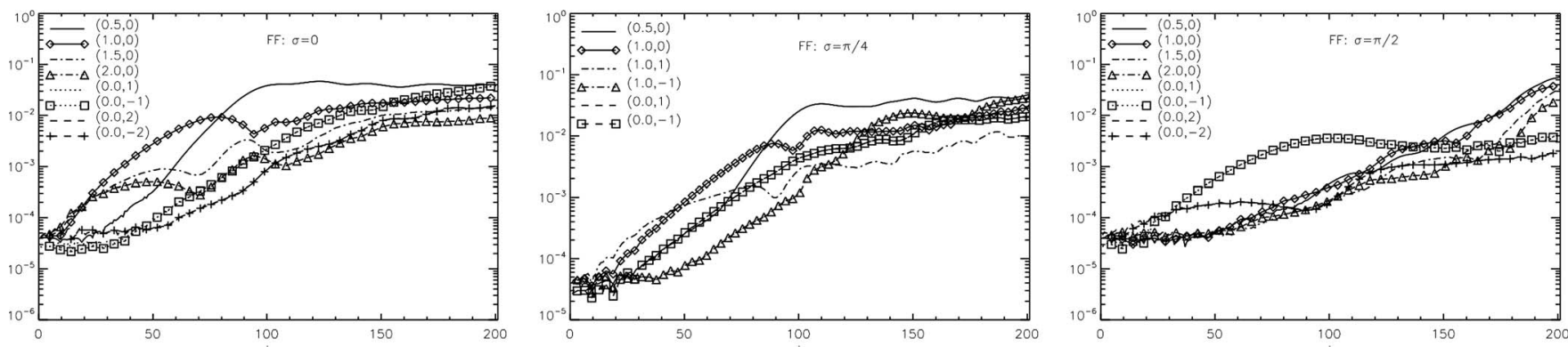

FIG. 5. From left to right, the most relevant Fourier modes of the magnetic energy in the nonlinear phase as a function of time for the FF configurations with $\sigma=0$ (left), $\sigma=\pi / 4$ (middle), and $\sigma=\pi / 2$ (right).

this case are those characterized by $k_{y}=1$ and $k_{z}=2,3$, corresponding to $|k|$ in the range $[\sqrt{5}, \sqrt{10}]$, which is consistent with the values predicted by the linear simulations for $\sigma=0$. Same results, not shown here, are obtained for $\sigma=\pi / 8$, and $\sigma=\pi / 4$. Moreover, the same results is obtained in the case of $\sigma=-\pi / 4$ (FF) configurations. This implies that the way the cross component of the magnetic field rotates through the current sheet is not relevant for the dynamics of the system.

As shown previously, in the two-dimensional case, for $\sigma=\pi / 2$, the system should be unstable under KH-like instability. However, if we look at the right panel of Fig. 4, we note that both FF and PB configurations are dominated by modes aligned in the $z$ direction, the direction of the AMF field, although a significant dose of energy is still present in modes with $k_{z}=0$ for the PB case. The most unstable modes lie in the wave-vector range $k_{z} \approx \pm 2-3$. The system is dominated by resistive-varicose instabilities. The presence of excited modes along the $y$ direction is due to the presence of the KH instability, stronger for the PB case because of the absence of the stabilizing effect of the perpendicular magnetic field inside the current sheet.
In conclusion, regardless of the $\sigma$ values, the most unstable modes driving the system throughout the linear regime are resistive-varicose and they are selected according to the AMF direction regardless of the detailed structure of the magnetic field within the current sheet, which is the type of equilibrium under consideration. In particular, these modes are characterized by the condition $k \times \mathbf{B}(|\delta x| \gg 1)=0$. Such preferential direction determined by the AMF in the linear evolution of the instability was observed also in the case of the incompressible and compressible 3D tearing mode evolution by Onofri et al. ${ }^{16}$ and Landi et al., ${ }^{17}$ respectively.

\section{B. Nonlinear regime}

Although the nonlinear regime of the current (neutral) double vortex sheet is characterized by a complex behavior, which depends on the initial evolution of the most unstable modes, it is possible to identify some key features.
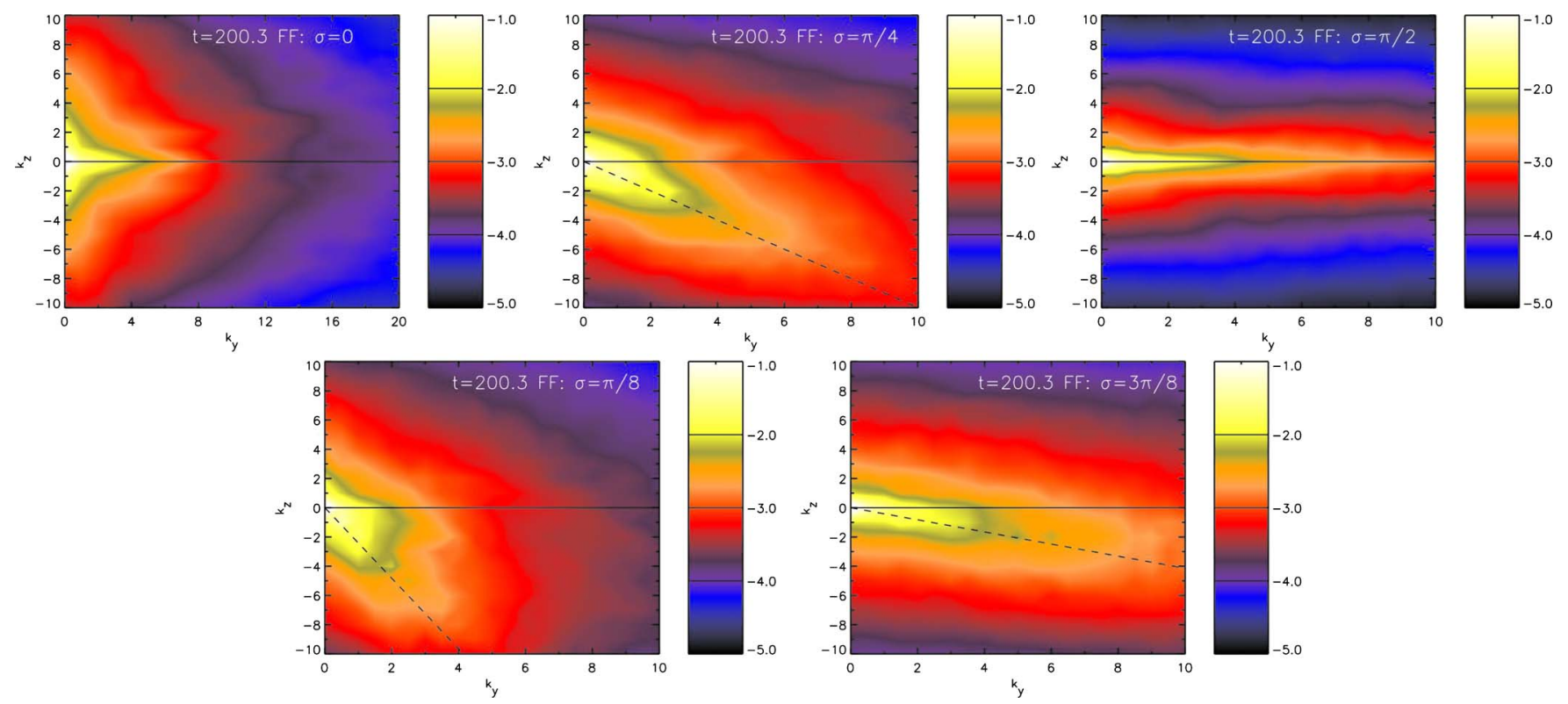

FIG. 6. (Color online) Magnetic energy in the $\left(k_{y}-k_{z}\right)$ Fourier space for the FF configurations corresponding to different values of $\sigma$ in the well-developed nonlinear regime. The dashed line highlights the $\sigma-\pi / 2$ direction. 


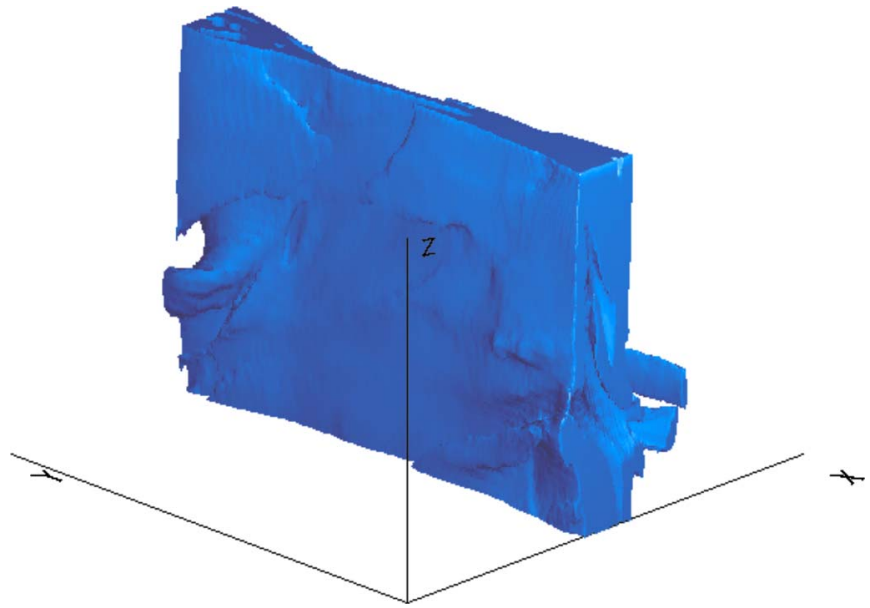

FIG. 7. (Color online) 3D plasma pressure isocontour at $t=200$ for the FF configuration with $\sigma=0$. The shaded surface encodes the region of space where the pressure is more than $65 \%$ of its maximum.

\section{FF cases}

We start from Fig. 5 to consider all the cases determined by a different value of $\sigma$. Here, we show the temporal evolution of some magnetic energy modes for three different FF configurations: $\sigma=0$ (left panel), $\sigma=\pi / 4$ (middle panel), and $\sigma=\pi / 2$ (right panel).

$\sigma=0$. From about $t \sim 50$, it is clearly observed a coalescence process driving the system to the maximum length scale allowed by our numerical domain. In fact, the inverse cascade ends as soon as the largest wave-number mode dominates at about $t \sim 80$. In the meanwhile, the orthogonal modes (those with $k_{y}=0$ and $k_{z} \neq 0$ ) keep on growing and, from $t \sim 150 \mathrm{on}$, they start to determine the structure of the system. At about $t \sim 200$, most of the energy is symmetrically distributed in the "secondary" (orthogonal) modes as well as in the first primary modes $\left(k_{y} \neq 0\right.$ and $\left.k_{z}=0\right)$, as shown in the top-left panel of Fig. 6, where the magnetic energy spectrum in the plane $\left(k_{y}, k_{z}\right)$ in the well-developed nonlinear regime is shown for different $\sigma$-valued FF initial configurations. This dynamics is analogous to those shown in Landi et al. $^{17}$ analyzing the 3D compressive tearing evolution. Also in that study, the nonlinear magnetic energy spectrum is characterized by two preferred directions resulting in a system modulated in two different ways in the physical space. In Fig. 7 it is shown the 3D plasma pressure isocontour for the " 0 " case at $t=200$ and it is possible to observe a coalesced plasma structure along the streamwise direction modulated along the spanwise axis.

$0<\sigma<\pi / 2$. The overall dynamics is not very different from the previous case, provided that the nonlinear driving modes are properly considered. For instance, in the " $\pi / 4$ " case shown in Fig. 5 (middle panel), we observe a coalescence process with about the same time scale of the " 0 " case. Afterwards, the modes with a given direction to the AMF starts to increase, in particular mode $(1,-1)$, and in the far nonlinear regime they own most of the system energy as shown in the top-middle panel of Fig. 6. In the physical space, the rise of the secondary instability determines that the globally coalesced structure observed in the left panel of Fig. 8 at about $t \sim 100$ at later times is modulated along the direction located at $\pi / 4$ with respect to the $x-y$ plane as shown in the middle and right panels of Fig. 8. In Fig. 6 we show also the magnetic energy spectra at the end of the nonlinear regime also for the cases $\sigma=\pi / 8$ (bottom-left panel) and $3 \pi / 8$ (bottom-right panel). The dashed lines represent the $\sigma-\pi / 2$ direction and they well fit the observed spectrum anistropy direction. In all cases, It is worth pointing out that this privileged direction at about $t \sim 200$ is orthogonal to the direction of the most energetic modes in the linear regime (see Fig. 2 as reference for the $3 \pi / 8$ case). The 3D plasma pressure isocontours at the end of the simulations for the $\sigma$ $=\pi / 8$ (left panel) and for $\sigma=3 \pi / 8$ case (right panel) shown in Fig. 9 reveal the effects in the physical space of the behavior observed in the magnetic energy spectra.

$\sigma=\pi / 2$. As for the previous cases, the first part of the nonlinear evolution is characterized by the growth of the primary instability along the AMF, that is now the spanwise direction $z$. At about $t=125$, the largest coalesced structure with respect to our numerical box in the $z$ direction is developed. As shown in the left panel of Fig. 10, in the physical space enhancements of the plasma pressure underlines the presence of magnetic islands, as it is typical of resistive driven instabilities. Yet, the modes orthogonal to the primary ones are still growing and they reach the same energy level at about $t=150$. So far, system's behavior is mostly analogous to the previously described cases, characterized all by an energy density almost equally distributed both in primary and secondary modes. Yet, the final part of the nonlinear

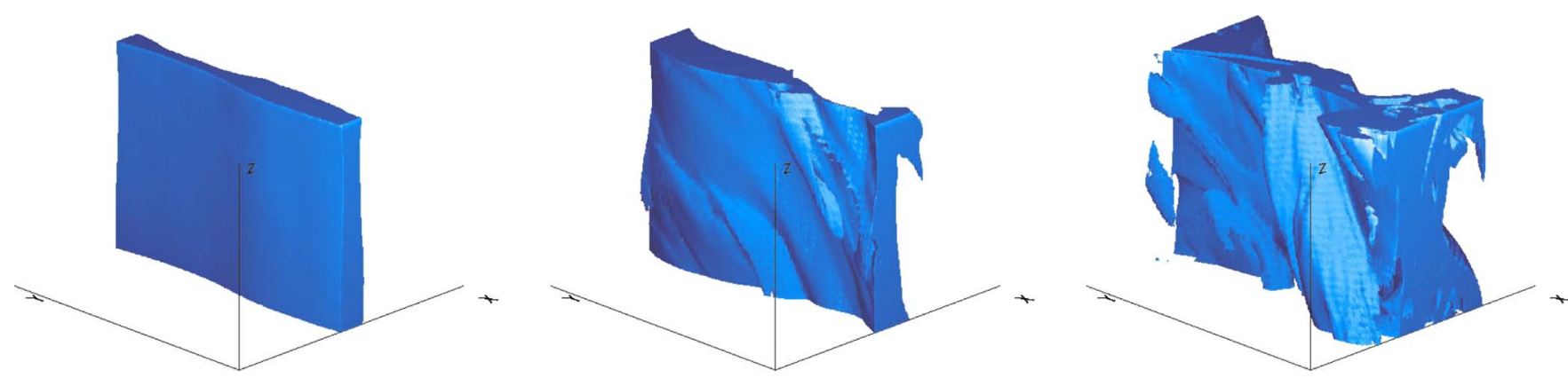

FIG. 8. (Color online) 3D plasma pressure isocontour at three different instants during the nonlinear evolution of the FF configuration with $\sigma=\pi / 4$. These images refer to $t=100$ (left panel), $t=150$ (middle panel), and $t=200$ (right panel). The shaded surface encodes the region of space where the pressure is more than $50 \%$ of its maximum. 

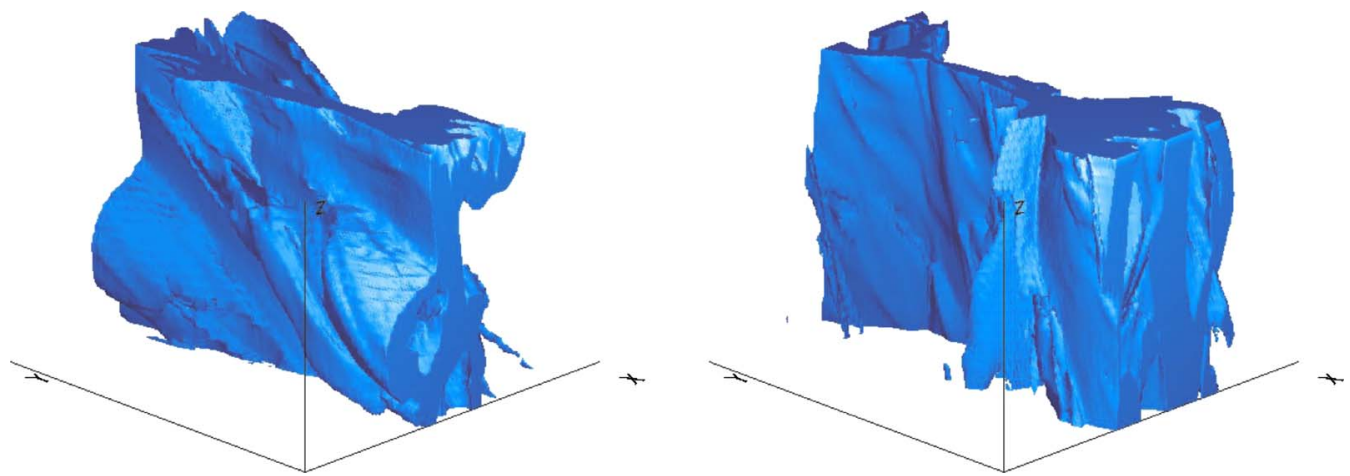

FIG. 9. (Color online) 3D plasma pressure isocontour at the end of the simulation ( $t=200)$ for two different FF configurations: $\sigma=\pi / 8$ (left panel) and $\sigma=3 \pi / 8$ (right panel). The shaded surface encodes the region of space where the pressure is more than $55 \%$ of its maximum.

evolution presents a completely different dynamics: the modes along the streamwise direction are dominating the others of about one order of magnitude in the energy spectrum (see Figs. 5 and 6). An analysis in the physical space reveals that the prevailing modes have a different nature with respect the previous cases. In fact, in the right panel of Fig. 10 , we observe that at $t=200$ the plasma pressure shows the typical sinuous profile of $\mathrm{KH}$ instabilities. So, although it is completely overwhelmed by the resistive-varicose mode in the linear stage, nevertheless the KH dynamics appears to dominate in the well-developed nonlinear regime and as it seems to be mainly driven by the presence of the velocity shear, we expect it to influence strongly the wake (jet) acceleration (deceleration) (see in Sec. III C).

So, as already mentioned, we observed a qualitatively similar behavior for different values of $\sigma<\pi / 2$. A resistive linear regime is driven by the modes selected according to the direction of the equilibrium AMF initially defined in the $(y, z)$ plane. The nonlinear regime is initially characterized by the formation of magnetic islands then undergoing a coalescence process. Afterwards, the onset of secondary instabilities kink the coalesced structures. In the later stages of the simulations, we observed plasma pressure enhancements strongly modulated by modes orthogonal to the primary modes, as similarly observed in $3 \mathrm{D}$ tearing simulations. ${ }^{17} \mathrm{In}$ the Fourier space, the presence of the secondary modes is evident in the magnetic density energy contained in modes orthogonal to the primary ones. For $\sigma=\pi / 2$, the system is also unstable to $\mathrm{KH}$ instabilities and this changes significantly its nonlinear dynamical path. In this case, the later stage of system's evolution is dominated by sinuous modes.

\section{2. $P B$ cases}

Now, we consider the nonlinear evolution of the PB equilibrium configurations for different values of $\sigma$. In Fig. 11, it is shown the time evolution of the most important magnetic energy modes for the cases $\sigma=0, \sigma=\pi / 4$, and $\sigma=\pi / 2$.

$\sigma=0$. We observe that at the beginning of the nonlinear regime the system is still driven by one of the linearly dominating modes, $\left(k_{y}, k_{z}\right)=(1,0)$, leading it to be structured almost on the largest length scale. In fact, after a few dozens of time steps, we observed an inverse cascade toward the maximum length scale allowed by our numerical box, $(0.5,0)$, followed by a saturation plateau of the nonlinear regime lasting up to about $t \sim 130$. In the meantime, the orthogonal modes $(0, \pm 1)$ and $(0, \pm 2)$ grow as well. So, the twodimensional structured system starts to develop in the $z$ direction according to these modes, which, together with their harmonics, drive the system until the end of the simulation. Primary modes directed along the $y$ direction, except for the fundamental one $(0.5,0)$, different from the FF configuration, are strongly damped. This is evident, for example, by comparing mode $(1.0,0)$ in Fig. 11, left panel, with the analogous one for the FF configuration in Fig. 5. So, from an early
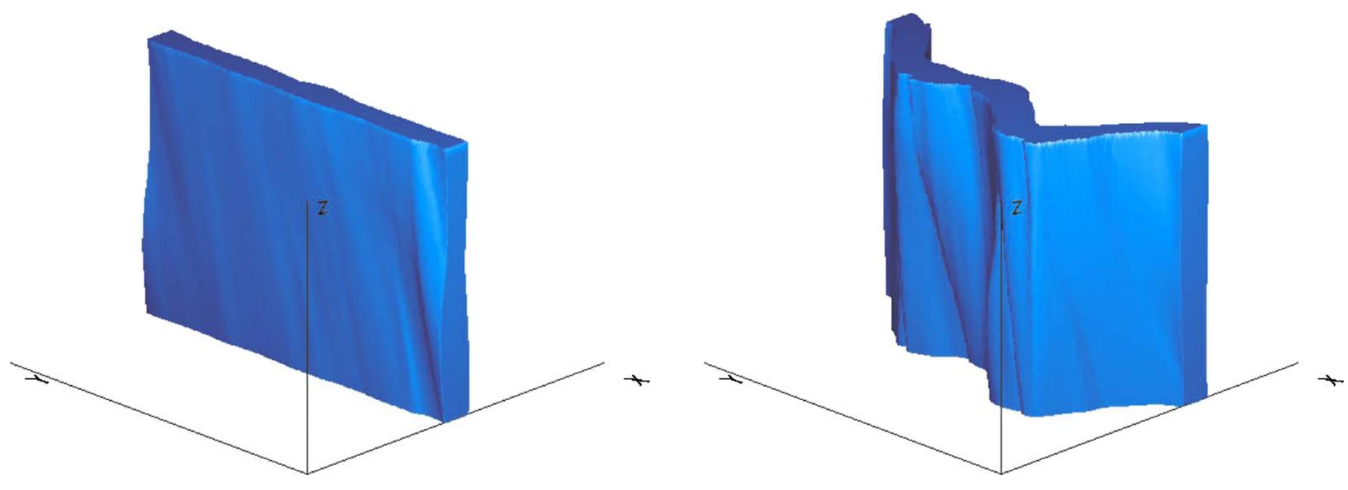

FIG. 10. (Color online) 3D plasma pressure isocontours of the FF configuration with $\sigma=\pi / 2$. The image refers to $t=125$ (left panel) and $t=200$ (right panel). The shaded surface encodes the region of space where the pressure is more than $50 \%$ of its maximum. 

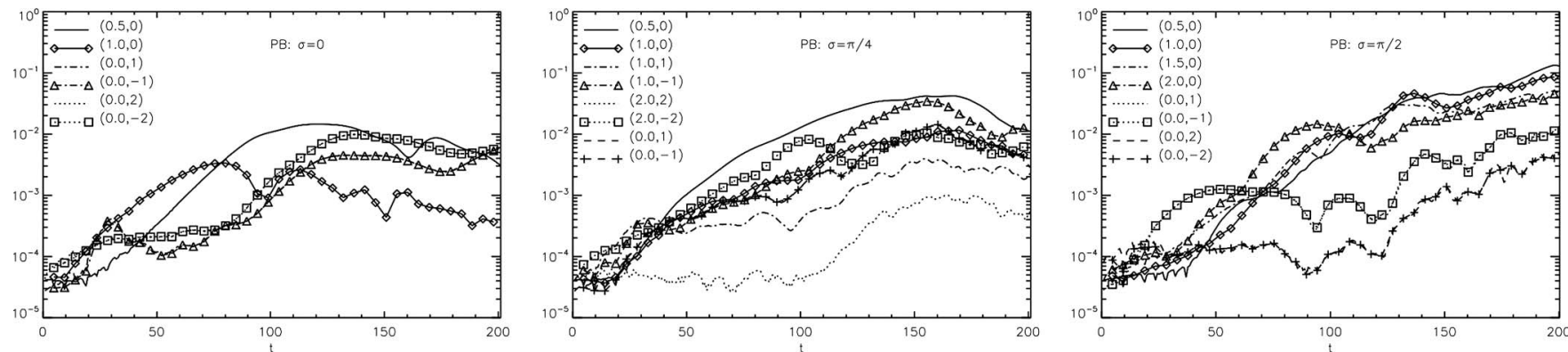

FIG. 11. From left to right, the most relevant Fourier modes of the magnetic energy in the nonlinear phase as a function of time for the PB configurations with $\sigma=0$ (left), $\sigma=\pi / 4$ (middle), and $\sigma=\pi / 2$ (right).

coalescing structure, the system presents at the end of the simulation an essentially two-dimensional configuration in the $(x, z)$, as observed in the plasma pressure structure shown in Fig. 12. This behavior is confirmed also by the magnetic energy spectrum at the end of the simulation, shown in the left panel of Fig. 13. Most of the magnetic energy is now contained in modes directed along the $z$ direction and we do not observe the energy redistribution between primary and secondary modes as in the analogous FF configuration. This result is consistent with those obtained in the pure $3 \mathrm{D}$ tearing instability simulations for the PB configuration. ${ }^{17}$

$0<\sigma<\pi / 2$. Let us focus on one emblematic case, such as $\sigma=\pi / 4$. After the first phase, where the system evolution is determined by the primary $3 \mathrm{D}$ modes characterizing the linear regime, as shown in the middle panel of Fig. 11, we have an increase in the contribution of the mode $\left(k_{y}, k_{z}\right)$ $=(0.5,0)$, which drives the dynamics until the end of the simulation. At the same time, modes orthogonal to the primary ones increase in time such that, at the end of the simulation, they contain a large portion of the magnetic fluctuating energy, as it is shown in the middle panel of Fig. 13. Again, the dashed line locates the $\sigma-\pi / 2$ direction and it well fits the observed spectrum anisotropy direction pointing out that the privileged direction at about $t \sim 200$ is orthogonal to the direction of the most energetic modes in the linear regime. A similar behavior is observed in the magnetic energy spectrum for the " $\pi / 8$ " and " $3 \pi / 8$ " cases (bottom-left and bottom-right panels of Fig. 13, respectively). The physical behavior of the system is shown in Fig. 12 (middle panel), where it is evident the instability modulation of the whole structure along the preferred direction. $\sigma=\pi / 2$. The overall behavior is similar to the corresponding FF configuration, although it appears faster in its evolution. At $t=50$, it is attained the saturation in the magnetic energy power in the smallest spanwise $(z)$ wave vector and it is observed a rapid growth of the energy of streamwise (y) modes. At $t=100$, the system is strongly structured in the $(x, y)$ plane with a typical varicose-mode configuration. However, the presence of $\mathrm{KH}$ instability modes leads the system to be structured in a typical sinuous configuration already at $t=150$ as it is possible to observe in the right panel of Fig. 12. At the end of the simulation, most of the magnetic energy is contained in streamwise $(y)$ wave vectors (Fig. 13, top right panel).

\section{Jet/wake evolution}

In general, a net acceleration effect along the streamwise direction of the jet/wake embedding the current sheet is observed, while the mean (positive and negative) velocity contributions along the other directions are negligible for almost all the nonlinear regimes. We can measure the net acceleration effect by averaging out the spanwise and streamwise dependences of the streamwise component of the velocity field, i.e., by looking at the function

$$
\left\langle v_{y}\right\rangle_{y z}=\frac{1}{L_{y} L_{z}} \int_{0}^{L_{y}} \int_{0}^{L_{z}} v_{y}(x, y, z) d y d z .
$$

In Fig. 14, we plot this quantity for different values of the angle $\sigma$ and for both FF and PB equilibria (first and second panel). For comparison, we report the same profiles for twodimensional simulations with the same initial condition in

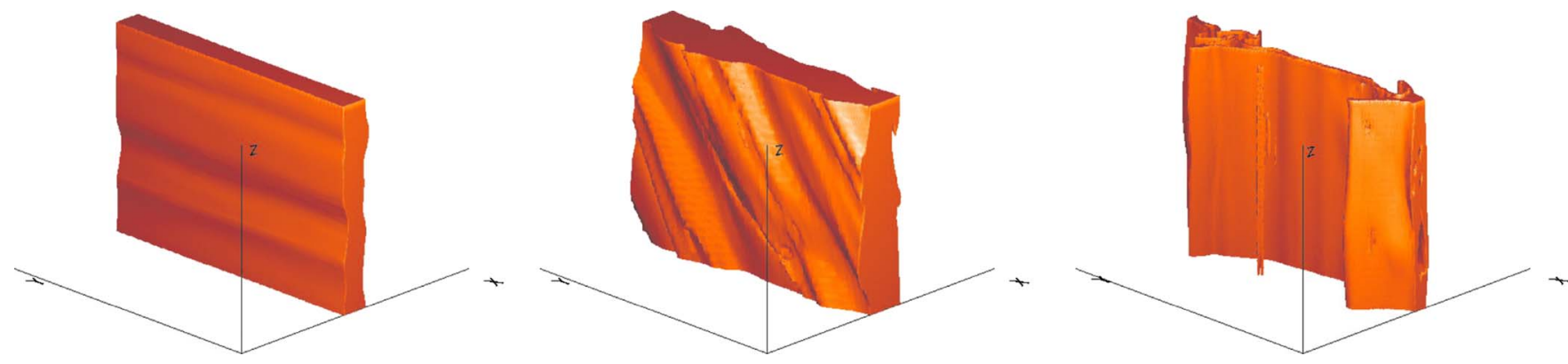

FIG. 12. (Color online) 3D plasma pressure isocontour during the nonlinear evolution of the PB configuration with $\sigma=0$ (left), $\sigma=\pi / 4$ (middle), and $\sigma=\pi / 2$ (right). The shaded surface encodes the region of space where the pressure is more than $60 \%$ of its maximum. For $\sigma=0$ and $\sigma=\pi / 4$ the pressure is evaluated at $t=200$, while for the $\sigma=\pi / 2$ the reference time is $t=150$. 

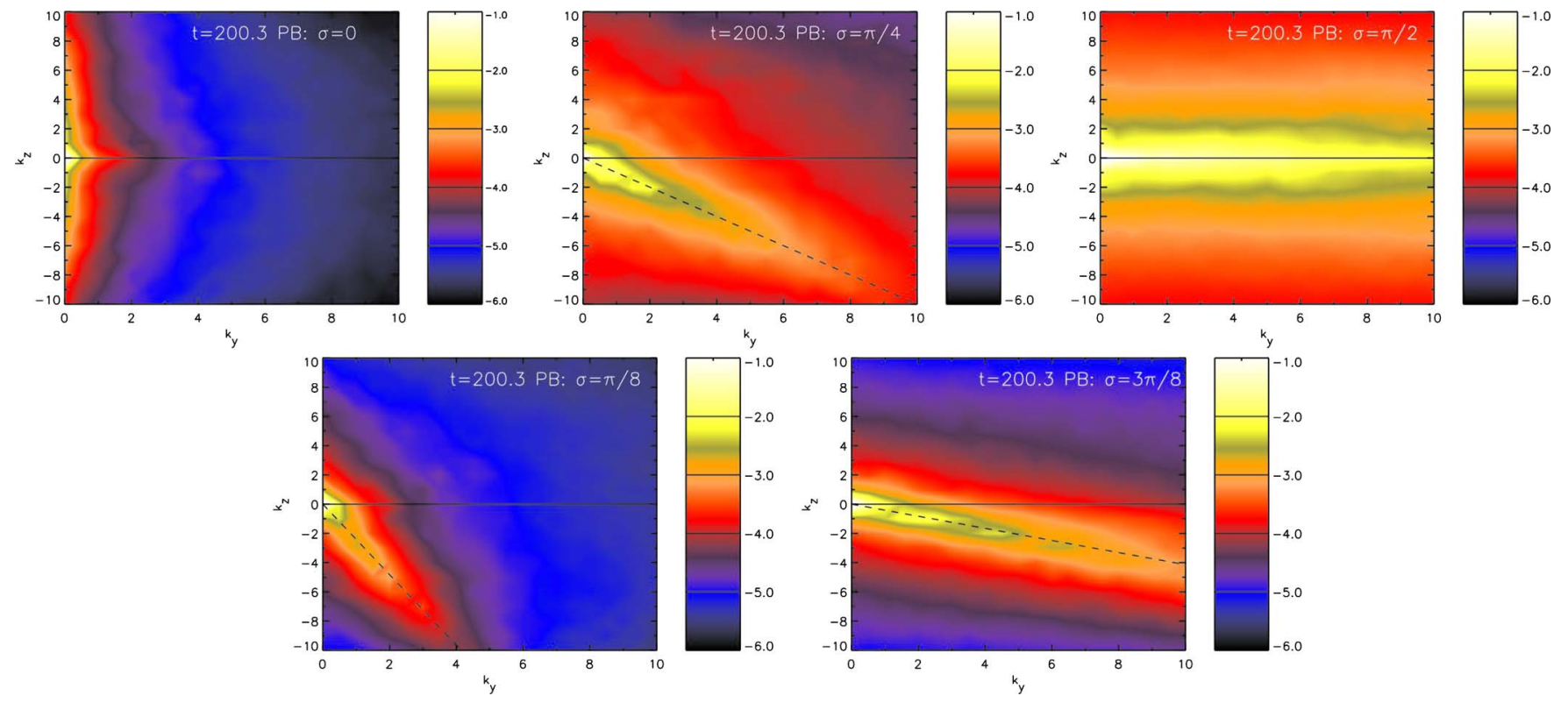

FIG. 13. (Color online) Magnetic energy in the $\left(k_{z}-k_{y}\right)$ Fourier space for the PB configurations with different values of $\sigma$ at $t \sim 200$. As for the FF case (Fig. 6), the dashed line highlights the angle $\sigma-\pi / 2$.

the well-developed nonlinear regime (third and fourth panel). It manifests the strong differences from the two-dimensional and the 3D case. Even though we are exploring a slightly different parameter space from Ref. 9, the two-dimensional simulations we performed confirms the overall dynamics discussed there. In three dimensions, we observed a weaker acceleration effect and a less pronounced enlargement of the wake, both in the FF and PB cases. Because of the insurgence of the secondary instability, dips in the velocity profiles observed in two-dimensional simulations, caused by the presence of coherent vortices structures inside the currentsheet region, are no longer present.

\section{DISCUSSIONS AND CONCLUSIONS}

We consider the fully 3D linear and nonlinear evolution of a plane neutral/current double vortex sheet consisting in a neutral/current sheet embedded in a sheared supersonic flow. Several parameters can influence the evolution of the initially perturbed system: the initial equilibrium configuration, the magnetic and velocity fields relative geometry, the plasma $\beta$, and the sonic and Alfvènic Mach numbers. Here we considered the typical values describing jet/wake flows in the solar active regions at a few solar radii; in particular, the configuration can be applied to the wake model of the slow component of the solar wind. ${ }^{7-9}$

Two different equilibrium configurations have been compared: a FF magnetic field whose polarity reversal determines the formation of a current sheet inside the plasma flow and a PB magnetic field where the polarity reversal is obtained by means of a neutral plane inside the flow. Different from the 2D simulations, ${ }^{9}$ for all orientations of the AMF, both the FF and PB equilibrium configurations are dominated by varicose-resistive modes. The most unstable ones are characterized by wave vectors whose direction is parallel to the AMF. The presence of a rotational component inside the current sheet, as for the FF case, does not affect significantly the linear behavior of the system. Such selection rules have been already observed in the previous analysis of $3 \mathrm{D}$ tearing mode evolution. ${ }^{17}$

The nonlinear regime is always characterized by a twostage evolution; for $\sigma<\pi / 2$, the varicose-resistive modes (primary modes) start to coalesce and then a secondary instability develops. In the physical space, it results in the formation of magnetic islands and pressure enhancement re-
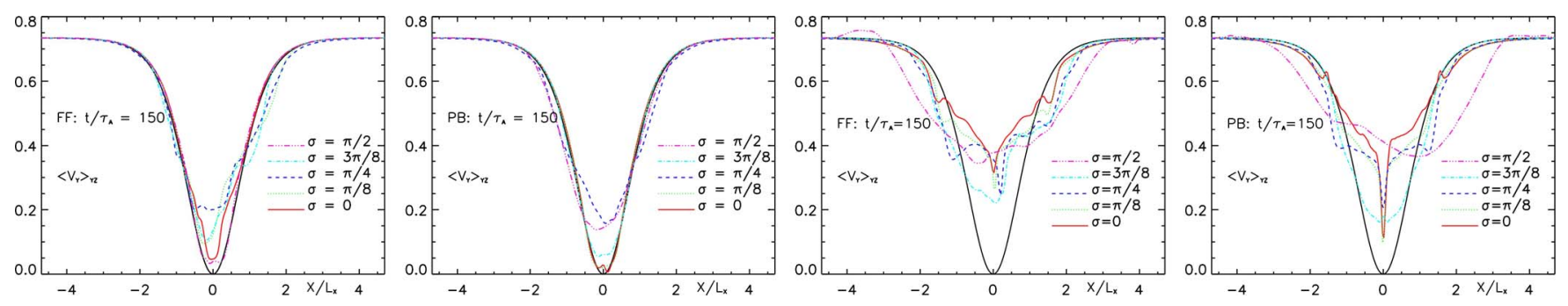

FIG. 14. (Color online) Wake profile of the streamwise component of the velocity, as computed using Eq. (22), for all the values of $\sigma$. The black solid line in all plots is the wake profile a $t=0$ shown as reference. The first and second panels refer, respectively, to the FF and PB 3D cases (see Sec. III C), whereas, for comparison, in the third and fourth panels it is reported the FF and PB two-dimensional cases, respectively. 

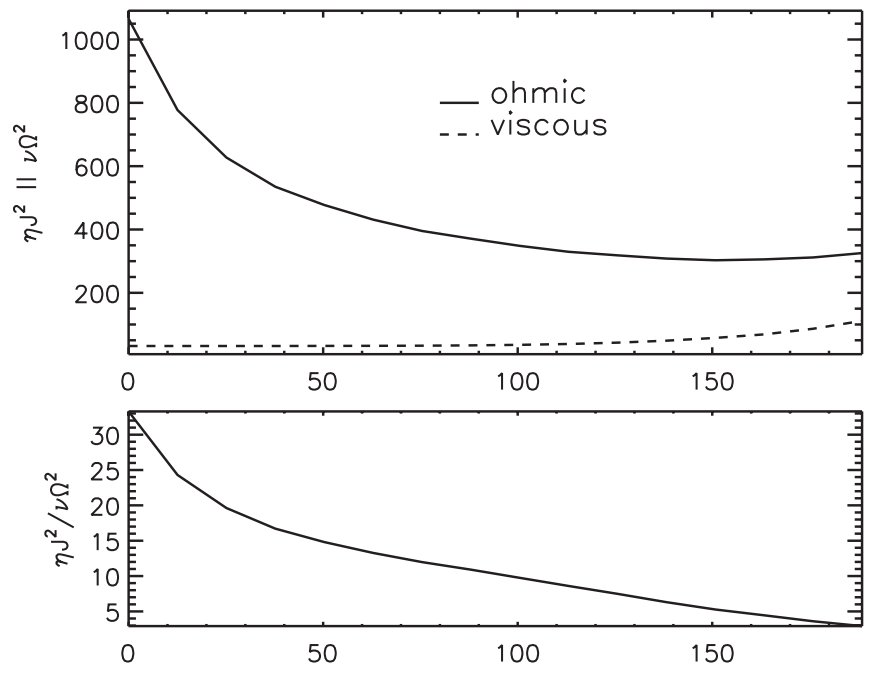

FIG. 15. Top panel: volume average of the Ohmic and viscous dissipation (solid and dashed lines, respectively) as a function of the simulation time for the FF simulation with $\sigma=\pi / 4$. Bottom panel: ratio of these quantities as a function of the simulation time.

gions, which afterwards are deformed by kinklike instabilities. The appearance of the secondary instability is accompanied by the growth of fluctuations whose wave vector are orthogonal to the primary ones. The resulting magnetic energy spectrum, as for the simple current-sheet instability, ${ }^{16,17}$ results thus strongly anisotropic. As pointed out by Landi et al., ${ }^{17}$ the presence of the magnetic field inside the current sheet in the FF configuration can reduce the effects of the secondary instability. As a consequence, in the fully developed nonlinear regime, a large dose of magnetic energy is in both the primary and secondary (orthogonal) modes. Because of the absence of a guide field, which has a stabilizing effect on the current-sheet evolution ${ }^{16,17,26}$ for the PB configuration, the transition to the secondary instability appears earlier and the magnetic energy spectrum is dominated by these modes. As in Battarini et al. ${ }^{9}$ the production of magnetic islands is accompanied by vortices formation, which are considered as responsible for the wake (jet) acceleration (deceleration). ${ }^{6,7}$ Similar to what was observed by Einaudi et al., ${ }^{6}$ the onset of the secondary instability determines the destabilization of the islands, hence it reduces the acceleration/deceleration effect.

For $\sigma=\pi / 2$, for sonic and Alfvénic Mach numbers typical of the heliospheric environment, the system results to be unstable both by tearing and $\mathrm{KH}$ instabilities. With the relatively low Lundquist number here adopted, it results that, differently from the two-dimensional case, ${ }^{9}$ in three dimensions, the linear regime is dominated by resistive instabilities. However, during the nonlinear regime, because of the dissipation of the underlying equilibrium magnetic field, the width of the current sheet increases and, as consequence, there is a decrease in time of the tearing mode growth rate as it goes as $\delta^{3 / 2} \cdot{ }^{27}$ As a consequence, if the diffusion acts for a sufficiently long time, the flow driven instability is able to dominate the system during the nonlinear regime. Moreover, since the growth rate of the resistive mode scales as $\mathcal{S}^{-1 / 2}$, it is expected that, for a magnetic configuration where the
AMF is orthogonal to the jet/wake, $\mathrm{KH}$ instabilities will be the prominent dynamics driving the overall system evolution. In the present work the current (neutral) double vortex sheet evolution is investigated in the limit of zero kinematic viscosity, in analogy with Ref. 17. In fact, although the influence of viscous effects can be retained with respect to the resistivity-driven dynamics, however, it has been suggested that the overall evolution depends essentially on the Hartmann number, which gives a measure of the relative importance of drag forces resulting from magnetic diffusivity and kinematic viscous forces. ${ }^{28}$ Figure 15 shows an a posteriori computation of the volumetric average of the Ohmic and the viscous dissipation (solid and dashed lines, respectively, in the top panel) and their ratio as a function of the simulation time. Here, a FF simulation with $\sigma=\pi / 4$ is considered, but this case is a paradigma for all simulations. The abovementioned quantities are defined, respectively, as

$$
\mathcal{W}_{\mathrm{ohm}}(t)=\int_{V} d V \eta|\mathbf{J}|^{2}
$$

$$
\mathcal{W}_{\text {vis }}(t)=\int_{V} d V \nu|\Omega|^{2},
$$

where we assume $\nu=\eta, \Omega$ is the vorticity vector, $\boldsymbol{\nabla} \times \mathbf{v}$, and $V$ is the volume of our $3 \mathrm{D}$ numerical box. In general, the overall Ohmic dissipation is far bigger than the viscous one: as the motion gets turbulent and vortices form and grow, the contribution of the viscosity becomes more and more important up to be one-fifth of the other. Yet, the overall dynamics throughout the linear and nonlinear regimes is not affected by that, in particular, with regards to the onset of the coalescence process or the triggering of ideal instabilities as the secondary ones. ${ }^{11}$ Nevertheless, a further investigation on viscosity effects in longer simulations is mandatory. For several heliospheric and astrophysical environment applications, it will be suitable to consider the effect of the radial expansion and/or the presence of an underlying stratified medium. They can influence not only the onset of the primary instability, but also the length- and time scales associated with the insurgence of the secondary instability.

\section{ACKNOWLEDGMENTS}

The authors are grateful to Luca Del Zanna and Andrea Verdini for the useful comments and suggestions. Numerical computations have been performed using the 512 processor IBM SP5 of the CINECA consortium available through the INAF-CINECA agreement 2006-2007: High Performance Computing Resources for Astronomy and Astrophysics. M. Velli was supported in part by the NASA LWS TR\&T program. This research was supported in part by ASI Contract No. I/015/07/0 "Solar System Exploration." We want to thank the IPAM program "The Grand Challenge Problems in Computational Astrophysics" at UCLA. We are also grateful to the anonymous referee for comments and suggestions. 
${ }^{1}$ L. Ofman, X. L. Chen, P. J. Morrison, and R. S. Steinolfson, Phys. Fluids B 3, 1364 (1991).

${ }^{2}$ S. Tsuneta, Astrophys. J. 456, 840 (1996).

${ }^{3}$ C. Uberoi, J. Geophys. Res. 89, 5652, DOI: 10.1029/JA089iA07p05652 (1984).

${ }^{4}$ Z. Y. Pu, M. Yei, and Z. X. Liu, J. Geophys. Res. 95, 10559, DOI: 10.1029/JA095iA07p10559 (1990).

${ }^{5}$ R. B. Paris and W. N.-C. Sy, Phys. Fluids 26, 2966 (1983).

${ }^{6}$ G. Einaudi, P. Boncinelli, R. B. Dahlburg, and J. T. Karpen, J. Geophys. Res. 104, 521, DOI: 10.1029/98JA02394 (1999).

${ }^{7}$ G. Einaudi, S. Chibbaro, R. B. Dahlburg, and M. Velli, Astrophys. J. 547, 1167 (2001).

${ }^{8}$ A. F. Rappazzo, M. Velli, G. Einaudi, and R. B. Dahlburg, Astrophys. J. 633, 474 (2005)

${ }^{9}$ L. Bettarini, S. Landi, F. A. Rappazzo, M. Velli, and M. Opher, Astron. Astrophys. 452, 321 (2006).

${ }^{10}$ R. B. Dahlburg, P. Boncinelli, and G. Einaudi, Phys. Plasmas 4, 1213 (1997).

${ }^{11}$ R. B. Dahlburg and G. Einaudi, Phys. Plasmas 8, 2700 (2001).

${ }^{12}$ R. B. Dahlburg and G. Einaudi, Phys. Plasmas 7, 1356 (2000).

${ }^{13}$ R. W. Metcalfe, S. A. Orszag, M. E. Brachet, S. Menon, and J. J. Riley, J. Fluid Mech. 184, 207 (1987).

${ }^{14}$ R. B. Dahlburg, S. K. Antiochos, and T. A. Zang, Phys. Fluids B 4, 3902 (1992).
${ }^{15}$ J. Schumacher and N. Seehafer, Phys. Rev. E 61, 2695 (2000).

${ }^{16}$ M. Onofri, L. Primavera, F. Malara, and P. Veltri, Phys. Plasmas 11, 4837 (2004).

${ }^{17}$ S. Landi, P. Londrillo, M. Velli, and L. Bettarini, Phys. Plasmas 15, 012302 (2008).

${ }^{18}$ R. B. Dahlburg, P. Boncinelli, and G. Einaudi, Phys. Plasmas 5, 79 (1998).

${ }^{19}$ P. Londrillo and L. Del Zanna, Astrophys. J. 530, 508 (2000).

${ }^{20}$ P. Londrillo and L. del Zanna, J. Comput. Phys. 195, 17 (2004).

${ }^{21}$ L. Del Zanna, N. Bucciantini, and P. Londrillo, Astron. Astrophys. 400, 397 (2003).

${ }^{22}$ L. Del Zanna, O. Zanotti, N. Bucciantini, and P. Londrillo, Astron. Astrophys. 473, 11 (2007).

${ }^{23}$ S. K. Lele, J. Comput. Phys. 103, 16 (1992).

${ }^{24}$ G. Mann, A. Klassen, H. Aurass, and H.-T. Classen, Astron. Astrophys. 400, 329 (2003).

${ }^{25}$ S. Chandrasekhar, Hydrodynamic and Hydromagnetic Stability (International Series of Monographs on Physics, Oxford, 1961).

${ }^{26}$ R. B. Dahlburg, J. A. Klimchuk, and S. K. Antiochos, Astrophys. J. 622, 1191 (2005).

${ }^{27}$ E. Priest and T. Forbes, Magnetic Reconnection (Cambridge University Press, Cambridge, 2000).

${ }^{28}$ R. B. Dahlburg, T. A. Zang, D. Montgomery, and M. Y. Hussaini, Proc. Natl. Acad. Sci. U.S.A. 80, 5798 (1983). 